
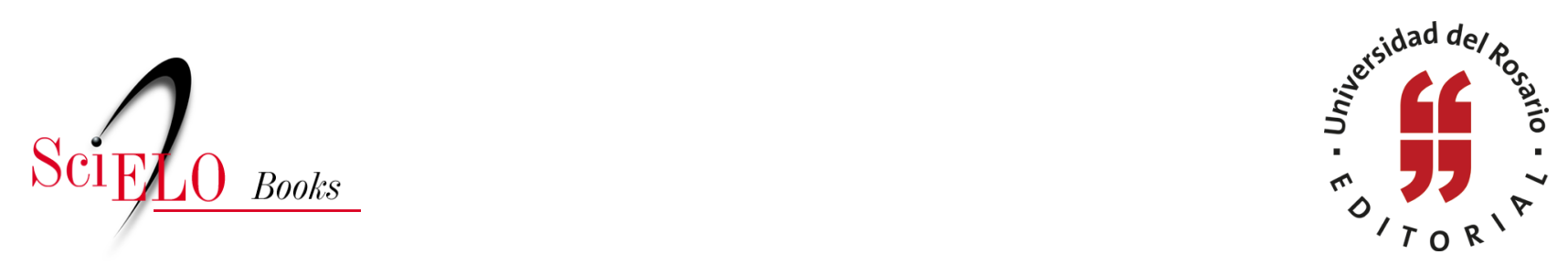

\title{
Reflexiones preliminares sobre la responsabilidad por daños ambientales
}

\author{
Gloria Amparo Rodríguez \\ Iván Vargas Chaves
}

AMPARO RODRÍGUEZ, G., and VARGAS CHAVES, I., ed. Reflexiones preliminares sobre la responsabilidad por daños ambientales. In: Perspectivas de responsabilidad por daños ambientales en Colombia [online]. Bogotá: Editorial Universidad del Rosario, 2015, pp. 1-11. Textos de Jurisprudencia collection. ISBN: 978-958-738-644-8. https://doi.org/10.7476/9789587386448.0001.

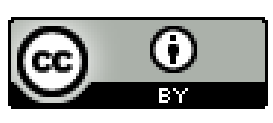

All the contents of this work, except where otherwise noted, is licensed under a Creative Commons Attribution 4.0 International license.

Todo o conteúdo deste trabalho, exceto quando houver ressalva, é publicado sob a licença Creative Commons Atribição 4.0.

Todo el contenido de esta obra, excepto donde se indique lo contrario, está bajo licencia de la licencia Creative Commons Reconocimento 4.0. 


\title{
Reflexiones preliminares sobre la responsabilidad por daños ambientales
}

\author{
Gloria Amparo Rodríguez \\ Iván Vargas Chaves
}

Hoy por hoy, es un hecho que como sociedad altamente globalizada e industrializada nos encontramos ante un entorno de deterioro ambiental difícilmente reparable; consecuencia de decisiones reflejadas en políticas que han menospreciando el lugar que ocupa el hombre en un medio que no le pertenece, y en donde han imperado los sistemas de producción y las nuevas tecnologías ante la conservación del ambiente de cara a las nuevas generaciones.

Este accionar humano se encuentra revestido por el poder de modificar la naturaleza atendiendo a los intereses propios, lo cual, de acuerdo con el principio de responsabilidad de Jonas (1985, p. 179), le habrá de imponer al hombre una carga u obligación ética orientada hacia el futuro. Se trata de un postulado que ha sido interpretado por diversos teóricos, entre ellos Attfield (1991, p. 202) y De Siqueira (2001, p. 280), como un llamado sensato que clama prudencia y equilibrio en las decisiones que advienen de la desconstrucción y la aleatoria reconstrucción tecnológica de la sociedad y el ambiente.

La razón por la cual esta obligación ética debe orientarse hacia el futuro se fundamenta en nuestra consideración en dos postulados. Primero, en el principio de igualdad intergeneracional como una extensión del derecho a la igualdad, ya que el derecho a la vida, así como otros derechos dentro del catálogo de libertades y garantías fundamentales, deben ser resguardados por la actual generación, tanto para sí misma como para las futuras generaciones. Y segundo, en los beneficios y cargas ambientales dentro de la dimensión distributiva del principio de la justicia ambiental.

En efecto, la justicia ambiental como una columna sobre los cimientos de la ética ambiental responde a una distribución equitativa de estas cargas, 
con miras a soportar las oportunidades de hacer parte de la dimensión participativa en la toma de decisiones de la igualdad intergeneracional (Ayestaran, 2008 , p. 156) y es en este punto en el que nuestros gobernantes tienen una gran tarea pendiente.

Jonas (1985, pp. 12-15), al reinterpretar el imperativo categórico de Kant, hace énfasis en la responsabilidad que tiene esta sociedad por ambas generaciones: la actual y la venidera, así como también para la biosfera en su conjunto. Una responsabilidad que aún no se ha asumido como es debido y que sigue llevándonos a todos los seres vivos a un escenario sombrío, amenazado por un desequilibrio ambiental que no tiene vuelta atrás.

No en vano secuelas como las alteraciones climáticas y ambientales han dado lugar a predicciones funestas sobre el desarrollo de toda la biosfera en general y a cuestionamientos sobre la supervivencia de las especies vivas en este planeta. Actualmente, incluso existe un amplio consenso por parte de la comunidad científica en relación con que el cambio climático es ya una realidad. Es el caso del Intergovernmental Panel on Climate Change, que desde 2007 viene reafirmando su postura sobre las actividades llevadas a cabo por el ser humano, las cuales seguirán alteranndo el panorama climático mundial.

Llegados aquí, el Banco Mundial y la Organización para la Cooperación y el Desarrollo Económico (oECD), entre otros organismos, plantearon, a través de un informe en la Convención Marco de las Naciones Unidas, un futuro en el que la alteración ambiental causada por el hombre: reducirá aún más el acceso al agua potable; afectará negativamente la salud de los habitantes de los países bajo el umbral de desarrollo; y se convertirá en una amenaza real para la seguridad alimentaria en muchos países de África, Asia y América Latina (Abeygunawardena et al., 2002, p. 7).

Para citar tan solo un ejemplo, en el informe sobre la economía del cambio climático en Paraguay, publicado por las Naciones Unidas a través de la Comisión Económica para América Latina y el Caribe (Cepal, 2014, p. 41), se prevén en dicho país reducciones significativas de la productividad de la agricultura familiar, con efectos sociales importantes; en el ámbito sanitario, se observaría un efecto diferenciado en patologías como dengue, malaria, enfermedades diarreicas e infecciones respiratorias agudas.

En cuanto a recursos hídricos, se afectaría la disponiblidad de agua de manera estacional, con una mayor variabilidad en las precipitaciones. Ello sin dejar a un lado los efectos de las subidas de temperatura, acompañadas de períodos 
de sequía más prolongados. Por último, destaca el referido informe que, si bien los impactos en la fauna y flora son difíciles de predecir, es claro que algunas especies podrían beneficiarse y otras extenguirse (Cepal, 2014, pp. 10-46-60).

De cualquier modo, los costes económicos y sociales de las afectaciones globales al ambiente son muy inciertos, pero quizá donde reside el mayor peligro es en el potencial de poner en riesgo el desarrollo no solo de los países pobres, sino de todos aquellos habitables por las generaciones venideras. Por ende, si bien algunos países con recursos logran mitigar los efectos globales, en un futuro a ninguno le será posible soportar ni la carga en términos de pérdida de vidas, ni los costes asociados a la devastación en el medio que traerán consigo las alteraciones ambientales.

Con todo y ello, no es necesario delinear una nueva visión del mundo, ya que el estado actual de la ciencia ha sido capaz de proveernos del real alcance de la naturaleza, como un desafortunado conjunto de objetos que el ser humano ha utilizado indiscriminadamente en las últimas décadas para satisfacer sus necesidades materiales, sin importar los efectos globales negativos, que de paso se han acelerado y que hoy en día ponen como un objeto de consideración ética la relación hombre-naturaleza.

Estamos ante lo que - para la concepción jonasiana - es fiel reflejo del divorcio entre los avances científicos y la reflexión ética, en tanto las nuevas tecnologías han introducido acciones, objetivos y consecuencias de magnitudes tan diferentes e imprevisibles que los marcos de la ética tradicional no son capaces de contener (De Siqueira, 2001, p. 281).

Adicional a lo anterior, como lo advierte Grandjean (2013, p. 627), la ciencia tampoco ha sido capaz de hacer un seguimiento adecuado a las decisiones en materia de políticas públicas, en pro del mejoramiento de las condiciones ambientales.

De acuerdo con la ética ontológica de Jonas (1985, p. 28), el hombre es parte del mundo en todos los aspectos y en este sentido ha adoptado decisiones apartadas de los intereses que le han de caracterizar como un ser que pertenece a lo que Wolsing (2014) denomina un mundo de la vida. Se trata de intereses que abarcan aspectos tales como la preservación, el bienestar y la autorrealización de la especie propia, así como de la vida en su concepción más amplia, lo cual a su vez depende de la asunción de obligaciones morales respecto a los demás. 
Pero ciertamente no hablamos del cumplimiento de dichas obligaciones cuando como especie vemos cómo hay más seres humanos en el mundo pasando hambre de lo que jamás hubo en la historia de la humanidad, como consecuencia de sequías, enfermedades o desastres naturales inducidos muchas veces por el hombre. Es el caso de la contaminación acuífera por la explotación de minerales a través de sustancias como el mercurio, para citar tan solo un caso que engloba este panorama.

Ahora bien, cuando se tiene una expectativa ante este panorama de que la población humana se duplicará en los siguientes siglos y de momento todo pareciera indicar que no podríamos dejarles a las generaciones venideras un patrimonio ambiental (De Siqueira, 2011, p. 99), inevitablemente nos surge la siguiente cuestión ¿qué podemos hacer ahora para cambiar esta proyección?

La respuesta, sin lugar a duda, podemos encontrarla una vez más en uno de los postulados de Jonas (1985, p. 11), en el que sostiene que el ser humano debe obrar de tal modo que los efectos de sus acciones sean compatibles con una vida auténtica y con la expectativa de continuar habitando este planeta.

Para comprender el concepto de vida auténtica en la perspectiva jonasiana, debemos referirnos a un tercer postulado que propone Ayestaran (2008, p. 155) sobre el principio de responsabilidad de Jonas, el cual, acompañando a los ya mencionados postulados de la igualdad intergeneracionaly de la justicia ambiental, propugna por la conservación de la integridad del ecosistema y de su biodiversidad. Nos referimos al postulado del respeto por la naturaleza.

En concordancia con este principio, el respeto parte del reconocimiento de axiomas tales como que la prosperidad de la raza humana depende de la prosperidad de la naturaleza; que de la supervivencia de la biósfera en todo su conjunto depende la supervivencia de las futuras generaciones; y que la economía humana es apenas un subsistema de la economía de la naturaleza, por lo que la primera debe encajar en esta, acatando sus leyes, y no aspirar a que la naturaleza sea forzosamente la que lo haga (Ayestaran, 2008, p. 156).

Desde la óptica del derecho, coincidimos con Ruda (2008) en que el gran problema aún no solucionado es el de la catalogación del ambiente como víctima en estricto sentido dentro del sistema de derecho civil en general, y de la responsabilidad civil en especial, así como dentro de otras disciplinas jurídicas. La razón, mientras que al derecho civil ni siquiera le interesan todas las relaciones interpersonales, la responsabilidad civil opera únicamente para aplicar una justicia conmutativa entre quien produce el daño y a quién le perjudica. 
Con lo anterior, queremos decir que, aunque en los últimas décadas la preocupación por el ambiente se ha incorporado en la instrumentación normativa a nivel internacional (García-Castellón, 2006, p. 506), es, en realidad, una preocupación que apenas ha logrado amortiguar los daños irreversibles causados por el hombre, pues el derecho tal y como lo conocemos en la actualidad es incapaz de enderezar la conducta humana hasta tanto no revista al ambiente la condición de víctima.

Para explicar este argumento, a través del deterioro ambiental difuso que afecta a recursos que no le pertenecen a nadie, Ruda (2008) se vale del relato del joven Parsifal y el cisne, que se encuentra en el primer acto del poema de Wolfram von Eschenbach, adaptado en 1887 por Richard Wagner en una de sus célebres óperas.

Parsifal dispara su flecha contra el cisne salvaje que se encontraba inocentemente en el lago, no daña la propiedad de nadie en particular, pero es obvio que destruye uno de los componentes del medio natural y que causa dolor a pesar a los moradores del lugar. En este supuesto, como en otros menos poéticos, la primera impresión es que no tiene sentido hablar de un dominus que soporte el daño o que pueda trasladarlo a otro, como se hace en los daños individuales, ya que no existe propiamente un titular perjudicado. Cabe, por ello, hacer al derecho de la responsabilidad la misma pregunta con que otro de los personajes de dicha ópera increpa al transgresor: el que fue nuestro cisne querido, ¿qué significa ahora para ti? (p. 33).

$\mathrm{Y}$ es que, si nos atenemos a las secuelas de las alteraciones ambientales severas, vemos cómo estas han sido más colectivas que individuales, como consecuencia de lo que, en términos de Ruda, es la interdependencia de los recursos naturales, integrados en un sistema único de solución de continuidad, o, lo que es lo mismo, el ecosistema (p. 34). Por ende, es insuficiente que la responsabilidad civil compense individualmente el daño toda vez que este es común y a razón de que no siempre es posible determinar las víctimas concretas afectadas por dichas alteraciones.

En esta misma línea, le han antecedido a Ruda autores como Benjamin (1998), Busnelli (1991) y Prieur (2004), quienes han buscado ampliar el tratamiento jurídico del daño ambiental a un contexto de pluralidad, pues sostienen 
que, más allá del resarcimiento del patrimonio o de la salud de un individuo, es lo colectivo - en tanto el daño ecológico se manifiesta en un sentido puro o público- lo que debe primar.

Otro asunto son los daños reflejo o por rebote, producto de las alteraciones individuales que se pudieron haber presentado y que, para los efectos del derecho de daños, pueden exigirse como daño emergente, lucro cesante e, incluso, como un daño moral (Delgado-Schneider, 2012, p. 49). Un tema de estudio propio que, a efectos de continuar con nuestra línea argumentativa, no se aborda en el presente texto.

Así, el daño ambiental en estricto sentido, o puro, es aquel que se causa o bien al ambiente, o bien a cualquiera de sus elementos, como una realidad autónoma de los valores ortodoxos asociados a la salud y al patrimonio (Delgado-Schneider, 2012, p. 50). En este sentido, podemos afirmar que su titularidad recae en la sociedad, representada por la comunidad afectada y por cada uno de sus miembros. Sociedad que por demás en las últimas décadas ha adquirido una consciencia social del respeto y clama por la salvaguarda al ambiente.

No obstante, es reseñable que a esta consciencia social le contraste de manera notable el estancamiento que en políticas públicas y en materia de responsabilidad civil se ha presentado, salvo en algunos honrosos y calificados esfuerzos, como bien lo apunta Hunter (2005, p. 26).

Es por lo anterior que, al ya referido postulado de Ruda de la insuficiente responsabilidad civil en los daños ambientales, nos sumamos desde la academia, haciendo un llamado de atención para que los esfuerzos e iniciativas normativas tengan en cuenta el principio de responsabilidad jonasiano.

Nos referimos a aquella ética que antepone la supervivencia de la generaciones venideras al impulso del ser humano por actuar contra la naturaleza; a intentar dominarla; a destruir los aspectos de la naturaleza que nos molestan; y apropiarnos selectivamente de lo que creemos nos producirá confort, placer y seguridad (Capriles, 1994). Esta ética le habrá de imponer al legislador un deber de cuidado hacia la naturaleza dada su vulnerabilidad.

Con la presente obra colectiva, la Especialización y la Línea de Investigación en Derecho Ambiental ${ }^{1}$ de la Facultad de Jurisprudencia reafirma ese

\footnotetext{
${ }^{1} \mathrm{El}$ presente texto hace parte del proyecto de investigación: Seguimiento a la actividad legislativa y avances del Derecho Ambiental de Colombia.
} 
llamado de atención desde la contribución de varios juristas colombianos, en el marco del diálogo permanente que desde la academia se da hacia el interior de la sociedad colombiana. Cada autor emprende un tema monográfico que en sí mismo es el reflejo de una problemática relacionada con el ámbito de la responsabilidad civil por daños ambientales. En tal sentido, para otorgarle una consistencia sistemática y lógica a este esfuerzo, el contenido fue estructurado de la siguiente forma.

En primer lugar, Fernando Andrés Pico Zúñiga realiza un acercamiento doctrinal al estudio del daño extrapatrimonial en la responsabilidad civil, a partir de las afectaciones ambientales, atendiendo no solo al ya cuestionado enfoque individual del daño ambiental, sino al enfoque colectivo, con miras a proteger directa e indirectamente la comunidad que se refleja en la naturaleza, así como los derechos presentes y futuros, de acuerdo con la defensa que ha de prestar el ordenamiento jurídico al ambiente.

En la misma línea, Gustavo Adolfo Ortega Guerrero y Tito Simón Ávila Suárez abordan la conceptualización del daño ambiental desde el punto de vista normativo según la evolución desde el derecho ambiental internacional, las disposiciones constitucionales en Colombia y los aspectos normativos de la legislación que desarrollan los instrumentos de regulación directa como el procedimiento sancionatorio ambiental.

Posteriormente, se analiza la conceptualización del daño ambiental que se ha venido desarrollando, comenzando con un ejercicio de jurisprudencia internacional comparada, para concluir con un cotejo de los avances de la jurisprudencia nacional; $y$, finalmente, se presentan algunas cuestiones dogmáticas del daño ambiental, las cuales han establecido su relación con otros conceptos relevantes, como impacto, contaminación y pasivo ambiental, en perspectiva de una teoría de responsabilidad ambiental que supere aquellas patrimonialistas desarrolladas desde la concepción civilista.

En el capítulo correspondiente al estudio de la causalidad adecuada a la imputación objetiva en la responsabilidad civil colombiana, con aplicación particular en la responsabilidad civil ambiental, Sergio Rojas Quiñones y Juan Diego Mojica Restrepo analizan la atribución de un resultado dañoso a partir de un juicio puramente material. En este texto, realizan un primer acercamiento a las teorías de causalidad utilizadas por la jurisprudencia entre el perjuicio irrogado y la actuación del agente, la cual sugieren puede variar o, 
a la vez, arrojar resultados que, a la luz de la equidad y la justicia e, incluso, del sentido común, llegan a resultar exagerados o contraintuitivos.

En ese sentido, es emprendida dicha problemática a fin de, previo recuento de las diferentes soluciones que se le han dado a lo largo de la historia y sus inconvenientes, presentar la que, según estos autores, es la teoría más adecuada para determinar la causalidad en cada caso, superando los defectos que doctrinalmente se le atribuyen a aquellas que la precedieron.

Con esta propuesta, el lector podrá evidenciar cómo, a partir de la diferenciación entre una imputación material y una imputación jurídica u objetiva, se logran resultados más acordes a la noción de justicia que subyace a la responsabilidad civil, que son especialmente aplicables en materias controversiales, como es la causalidad en la responsabilidad civil ambiental. De ahí que el capítulo contenga un importante énfasis en este tema, que se tratará de manera protagónica.

A continuación, Jouline Ropero Patarroyo lleva a cabo un estudio de la cobertura del derecho civil colombiano en los daños por contaminación de hidrocarburos persistentes en el mar. Para ello, luego de examinar el contenido de la actual regulación de la responsabilidad civil en esta materia en Colombia, propone una serie de reflexiones respecto a si esta resulta o no suficiente para resarcir la totalidad de daños que se generan a partir de estos sucesos.

Es de destacar que su planteamiento gira en torno a que dicha regulación, soportada en el régimen internacional de la responsabilidad civil por daños derivados del derrame de hidrocarburos en el mar acogido por Colombia, resulta insuficiente para obtener la reparación de la totalidad de los daños efectivamente causados. Por lo cual afirma que es el juez civil quien debe asumir la facultad de ordenar la reparación de los daños no cubiertos, en desarrollo de los mecanismos disponibles en el derecho civil.

En el capítulo correspondiente a la facultad de prevención en el derecho sancionatorio administrativo ambiental, contenida en la Ley 1333 de 2009, los autores, Andrés Gómez Rey, Gloria Amparo Rodríguez e Iván Vargas Chaves, estudian esta figura con el fin de verificar cómo el derecho ambiental ha venido construyendo una dogmática y reglas propias, escapando de la tradición del derecho administrativo.

Lo anterior con miras a avanzar en el establecimiento y demostración de la forma en la cual el derecho ambiental está creando la dogmática propia necesaria para lograr su independencia y consolidación como un área autónoma 
de la disciplina jurídica. Al respecto, en este ejercicio de reflexión, es posible ver cómo la figura, aparte de estar diseñada por fuera de la teoría general del acto administrativo, también posee particularidades que la hacen única dentro del ordenamiento jurídico colombiano.

La última sección, a cargo de José López Oliva, se dedica al tratamiento del seguro ecológico dentro del régimen de la responsabilidad ambiental en Colombia. Una figura en la cual el legislador ha dispuesto su obligatoriedad, en tanto la actividad de explotación de un recurso o la realización de una obra o actividad requieran de una licencia o permiso ambiental.

Ello, teniendo presente una realidad y es que las empresas o personas naturales en el ejercicio de dicha actividad pueden generar daños al ambiente; $\mathrm{y}$, en dicho caso, se hace más que necesario un mecanismo en el que el potencial victimario tenga la posibilidad de trasladar el riesgo inherente a una compañía aseguradora. En este sentido, creemos que, más allá del propósito de salvaguardar su patrimonio, lo que fundamenta esta figura es la garantía misma de reparación del daño ambiental causado.

Como puede observarse, los aportes que son abordados en esta obra colectiva tienen su propia complejidad dentro del estado del arte del derecho ambiental en nuestro país y, por ende, merecen ser estudiados de forma puntual con la finalidad de facilitar su integral comprensión. Vale la pena resaltar que el compromiso de los autores que nos acompañan se refleja en su punto de vista respecto a cada tema particular, buscando con ello contribuir con el estudio de la problemática de los daños ambientales, a propósito del diálogo que al inicio propusimos. Bienvenido el debate al cual hacemos esta contribución desde el desarrollo de los objetivos de la Línea de Investigación en Derecho Ambiental.

\section{Bibliografía}

Abeygunawardena, P., Vyas, Y., Knill, P., Foy, T., Harrold, M., Steele, P., et al. (2002). Poverty and climate change: reducing the vulnerability of the poor through adaptation. Consultation draft presented at the Eighth Conference of Parties to the United Nations Framework Convention on Climate Change, United Nations, New Delhi.

Attfield, R. (1991). The ethics of environmental concern. Athens, GA: University of Georgia Press. 
Ayestaran, I. (2008). The second Copernican revolution in the Anthropocene: an overview. Revista Internacional de Sosteniblidad, Tecnología y Humanismo, 3, 145-157.

Benjamin, A. (1998). A responsabilidade civil pelo dano ambiental no direito brasileiro e as lições do direito comparado. Revista Roma e America, 6, 129-130.

Busnelli, F. (1991). Illecito civile: estratto dall' aggiornamento I dell'Enciclopedia giuridica. In Enciclopedia giuridica. Roma: Istituto Della Enciclopedia Italiana nell'Enciclopedia 'Giovanni Treccani'.

Capriles, E. (1994). Individuo, sociedad, ecosistema. Mérida (Venezuela): Consejo de Publicaciones de la Universidad de los Andes.

De Siqueira, J. (2001). El principio de responsabilidad de Hans Jonas. Acta Bioethica, 7(2), 277-285.

De Siqueira, J. (2011). Análisis crítico del artículo del prof. Jan Helge Solbakk en el prisma del principio de responsabilidad. Revista Redbioética Unesco, 2(4), 96-100.

Delgado-Schneider, V. (2012). La responsabilidad civil extracontractual por el daño ambiental causado en la construcción u operación de las carreteras. Revista de Derecho, 25(1), 47-76.

García-Castellón, J. (2006). Panorama de la responsabilidad internacional por daño ambiental. In M. Carmona-Lara \& L. Hernández Meza, Temas selectos de derecho ambiental (pp. 407-508). Ciudad de México: Universidad Nacional Autónoma de México.

Grandjean, P. (2013). Science for precautionary decision-marking. In EEA, Late lessons from early warnings: science, precaution, innovation (pp. 635-642). Luxembourg: Publications Office of the European Union.

Hunter, I. (2005). La culpa con la ley en la responsabilidad civil ambiental. $R e-$ vista de Derecho, 18(2), 9-25.

IPCC. (2007). Fourth assessment report: climate change. Intergovernmental Panel on Climate Change, IPCC Secretariat, Geneva. Recuperado de http://www. ipcc.ch/pdf/assessment-report/ar4/syr/ar4_syr.pdf

Jonas, H. (1985). The imperative of responsibility: in search of an ethics for the technological age. Chicago: University of Chicago Press.

Prieur, M. (2004). Droit de l'environnement. Paris: Dalloz. Ruda, A. (2008). El daño ecológico puro. Madrid: Thomson Aranzadi. 
United Nations Economic Commission for Latin America and the Caribbean (Cepal). (2014). La economia del cambio climático en el Paraguay LC/W.617 - 14-20018. Santiago de Chile: un-Cepal.

Wolsing, P. (2014). ¿Responsibility to nature? Nordicum-Mediterraneum, Icelandic Journal of Nordic and Mediterranean Studies, $8(3)$. 